\title{
A New Eighth Order Implicit Block Algorithms for the Direct Solution of Second Order Ordinary Differential Equations
}

\author{
Ademola M. Badmus \\ Department of Mathematics, Nigerian Defence Academy, Kaduna, Nigeria \\ Email: ademolabadmus@gmail.com
}

Received 7 August 2014; revised 11 September 2014; accepted 19 September 2014

Copyright @ 2014 by author and Scientific Research Publishing Inc.

This work is licensed under the Creative Commons Attribution International License (CC BY). http://creativecommons.org/licenses/by/4.0/

\section{c) (i) Open Access}

\begin{abstract}
This paper focuses on derivation of a uniform order 8 implicit block method for the direct solution of general second order differential equations through continuous coefficients of Linear Multistep Method (LMM). The continuous formulation and its first derivatives were evaluated at some selected grid and off grid points to obtain our proposed method. The superiority of the method over the existing methods is established numerically.
\end{abstract}

\section{Keywords}

Uniform Order, Second Order Initial Value Problem, Implicit Block Algorithms, Zero Stable

\section{Introduction}

In the past, efforts have been made by many researchers to develop an efficient algorithm for solving second order differential equations of the form

$$
y^{\prime \prime}=f\left(x, y, y^{\prime}\right), y(0)=\alpha, y^{\prime}(0)=\beta
$$

directly through the interpolation and collocation points (see [1]-[4] to mention a few). Since many numerical techniques are available for the solution of higher order initial value problems (IVPs) and these techniques depend on many factors such as speed of convergence, computational expenses, data storage requirement and accuracy.

This paper aimed to address all these factors in the process of derivation and the implementation of this new 
method. Seven-point solutions are obtained from the block at once which speed up the computational processes; the method is self-starting and we obtained better accuracy over the existing methods.

The Equation (1.0) where $f$ is a continuous function, is conventionally solved by first reducing it to a system of first order differential equations and then applying the various first order methods available for their solutions. This approach is extensively discussed and established by some of the following researchers such as ([5] [6]). Also [7] [8] and [9] showed that this approach was associated with certain drawbacks. Due to the dimension of the problem after it has been reduced to a system of first order ordinary differential equations (ODEs), also the reduced systems of ODEs are not well posed unlike the given problem. The approach wastes a lot of computer time and human efforts, hence there is a great need to develop new and efficient algorithms to handle problem (1.0) directly without any reduction to its equivalent system of first order ODEs.

Several authors have also solved problem (1.0) through predictor corrector mode (PC) of implementations; among them are [10] and [11]. Although the implementation of the methods in a PC mode yields good accuracy, the approach is more costly to implement, for instance PC routines are very complicated to write, since they require special techniques for supplying starting values and also predicting all the off grid points present in the method which leads to longer computer time and human efforts to handle their approach.

In our new algorithms, we take great advantage of this approach by exploring its continuous formulation nature to obtain some discrete schemes when evaluated at some $x_{n+j}, j=[0, k]$ to form our block method; schemes are equally obtained from the derivative of the continuous formula.

\section{Definition 1.0}

A linear multi-step method is said to be zero-stable if the roots $R_{j}, j=1(1) k$ of the first characteristic polynomials

$$
\rho(R)=\operatorname{det}\left[\sum_{i=0}^{k} A_{i} R^{k-i}\right]=0, A_{0}=-1, \text { satisfies }\left|R_{j}\right| \leq 1 .
$$

If one of the roots is +1 , we call this the principal root of $\rho(R)$ (see [12]).

\section{Definition 1.1}

A linear multi-step method

$$
y(x)=\sum_{j=0}^{k} \alpha_{j}(x) y_{n+j}=h^{2} \sum_{j=0}^{k} \beta_{j}(x) f_{n+j} .
$$

We associate the linear differential operator

$$
L[y(x) ; h]=\sum_{j=0}^{k}\left[\alpha_{j} ; y(x+j h)-h^{2} \beta_{j} y^{\prime \prime}(x ; j h)\right]
$$

where $y(x)$ is an arbitrary function, continuity differentiable on $[a, b]$.

Expanding the test function $y(x+j h)$ as Taylor series about $x$ and collecting terms gives

$$
L[y(x) ; h]=C_{0} y(x)+C_{1} h y^{\prime}(x)+\cdots+C_{q} h^{q} y^{q}(x)+\cdots
$$

where $C_{0}, \cdots, C_{q}$ are constants.

A simple calculation yields the following formulae for the constants $C_{q}$ in terms of the coefficients $\alpha_{j}, \beta_{j}$.

$$
\begin{aligned}
C_{0} & =\alpha_{0}+\alpha_{1}+\alpha_{2}+\alpha_{3}+\cdots+\alpha_{k} \\
C_{1} & =\alpha_{1}+2 \alpha_{2}+3 \alpha_{3}+\cdots+k \alpha_{k} \\
C_{2} & =\frac{1}{2 !}\left(\alpha_{1}+2^{2} \alpha_{2}+3^{2} \alpha_{3}+\cdots+k^{2} \alpha_{k}\right)-\left(\beta_{0}+\beta_{1}+\beta_{2}+\cdots+\beta_{k}\right) \\
& \vdots \\
C_{q} & =\frac{1}{q !}\left(\alpha_{1}+2^{q} \alpha_{2}+3^{q} \alpha_{3}+\cdots+k^{q} \alpha_{k}\right)-\frac{1}{(q-2) !}\left(\beta_{1}+2^{q-2} \beta_{2}+\cdots+k^{q-2} \beta_{k}\right) \quad q=2,3, \cdots .
\end{aligned}
$$

Hence, we say that the method has order $P$ if $C_{0}=C_{1}=C_{2}=C_{p}=C_{p+1}=0$, but $C_{p+2} \neq 0$. Then $C_{p+2}$ is the error constant and $C_{p+2} h^{p+2} y^{p+2}\left(x_{n}\right)$ is the principal local truncated error at the point $x_{n}$. 


\section{Theory of Block Methods for Second Order Initial Value Problems}

Within the $r$-vectors $y_{m}$ and $f_{m}$ (for $\left.m=n r, n=0,1, \cdots\right) \quad y_{m}=\left(y_{n+1}, y_{n+2}, y_{n+3}, \cdots, y_{n+r}\right)^{\mathrm{T}}$

$$
f_{m}=\left(f_{n+1}, f_{n+2}, f_{n+3}, \cdots, f_{n+r}\right)^{\mathrm{T}} \text {. }
$$

The $S$ block $r$-point methods for

$$
\sum_{j=0}^{k} \alpha_{j}(x) y_{n+j}=h^{2} \sum_{j=0}^{k} \beta_{j}(x) f_{n+j}
$$

are given by the matrix finite difference equation.

$$
A^{0} y_{m}=\sum_{j=0}^{k} A^{(i)} y_{m-i}+h^{2} \sum_{j=0}^{k} B^{(i)} f_{m-i}
$$

where $A^{(i)}, B^{(i)}, i=0,(0)$ are $r \times r$ matrices respectively with element $a_{i j}^{(i)}, b_{i j}^{(i)}$, for $i j=0(1) r$.

The block scheme (2.0) is explicit if the coefficient matrix $B^{0}$ is a null matrix.

Let

$$
Z_{n}=\left(\begin{array}{c}
y\left(x_{n+1}\right) \\
y\left(x_{n+2}\right) \\
\vdots \\
y\left(x_{n+r}\right)
\end{array}\right),
$$

be respectively the theoretical solution to Equation (1.0) (see [12] [13]).

\section{Specification of the Method}

We consider a power series of single variables $x$ in the form

$$
P(x)=\sum_{j=0}^{\infty} \alpha_{j} x^{j}
$$

which is used as the basis or trial function to produce our approximate solution to (1.0) as

$$
\begin{gathered}
P(x)=\sum_{j=0}^{m+t-1} \alpha_{j} x^{j} \\
P^{\prime}(x)=\sum_{j=1}^{m+t-1} j \alpha_{j} x^{j-1} \\
P^{\prime \prime}(x)=\sum_{j=2}^{m+t-1} j(j-1) \alpha_{j} x^{j-2}=f\left(x, y, y^{\prime}\right)
\end{gathered}
$$

where $a_{j}$ are the parameters to be determined, $t$ and $m$ are point of interpolation and collocation points. The Equation (3.3) is collocated at $x=x_{n+j}, j=(0, k)$ and interpolating (3.1) at $x=x_{n+j} j=0, \frac{1}{2}$, with this method $k=3$ and specifically gives the following system of non linear equations of the form $h$

$$
\begin{gathered}
\sum_{j=0}^{m+t-1} \alpha_{j} x^{j}=y_{n+i}, \quad i=\left[0, \frac{1}{2}\right] \\
\sum_{j=2}^{m+t-1} j(j-1) \alpha_{j} x^{j-2}=f_{n+i}, \quad i=\left[0, \frac{1}{2}, \frac{3}{4}, 1, \frac{3}{2}, 2, \frac{5}{2}, 3\right] .
\end{gathered}
$$

The continuous formulation of the method will be of the form 


$$
\begin{aligned}
y(x) & =\alpha_{n} y_{n}+\alpha_{n+\frac{1}{2}} y_{n+\frac{1}{2}} \\
& =h^{2}\left[\beta_{n} f_{n}+\beta_{n+\frac{1}{2}} f_{n+\frac{1}{2}}+\beta_{n+\frac{3}{4}} f_{n+\frac{3}{4}}+\beta_{n+1} f_{n+1}+\beta_{n+\frac{3}{2}} f_{n+\frac{3}{2}}+\beta_{n+2} f_{n+2}+\beta_{n+\frac{5}{2}} f_{n+\frac{5}{2}}+\beta_{n+3} f_{n+3}\right] .
\end{aligned}
$$

When using Maple 17 mathematical software to obtain the values of $\alpha_{j} s$ in (3.4), (3.5) and substituting the values in Equation (3.0) to obtain our continuous formulation in the new method as

$$
\begin{aligned}
& y(x)=\frac{\left(h-2\left(x-x_{n}\right)\right)}{h} y_{n}+\frac{2\left(x-x_{n}\right)}{h} y_{n+\frac{1}{2}}+\left[-\frac{1115837 h}{10886400}\left(x-x_{n}\right)+\frac{1}{2}\left(x-x_{n}\right)^{2}-\frac{1683}{1620 h}\left(x-x_{n}\right)^{3}\right. \\
& +\frac{420}{324 h^{2}}\left(x-x_{n}\right)^{4}-\frac{5453}{5400 h^{3}}\left(x-x_{n}\right)^{5}+\frac{339}{810 h^{4}}\left(x-x_{n}\right)^{6} \\
& \left.-\frac{413}{2835 h^{5}}\left(x-x_{n}\right)^{7}+\frac{45}{1890 h^{6}}\left(x-x_{n}\right)^{8}-\frac{2}{1215 h^{6}}\left(x-x_{n}\right)^{9}\right] f_{n} \\
& +\left[-\frac{313243 h}{604800}\left(x-x_{n}\right)+\frac{270}{45 h}\left(x-x_{n}\right)^{3}-\frac{1143}{90 h^{2}}\left(x-x_{n}\right)^{4}+\frac{957}{75 h^{3}}\left(x-x_{n}\right)^{5}\right. \\
& \left.-\frac{325}{45 h^{4}}\left(x-x_{n}\right)^{6}+\frac{740}{315 h^{5}}\left(x-x_{n}\right)^{7}-\frac{43}{105 h^{6}}\left(x-x_{n}\right)^{8}+\frac{4}{135 h^{6}}\left(x-x_{n}\right)^{9}\right] f_{n+\frac{1}{2}} \\
& +\left[\frac{96496 h}{127575}\left(x-x_{n}\right)-\frac{92160}{8505 h}\left(x-x_{n}\right)^{3}+\frac{32256}{1215 h^{2}}\left(x-x_{n}\right)^{4}-\frac{59392}{2025 h^{3}}\left(x-x_{n}\right)^{5}\right. \\
& \left.+\frac{21504}{1215 h^{4}}\left(x-x_{n}\right)^{6}-\frac{51200}{8505 h^{5}}\left(x-x_{n}\right)^{7}+\frac{3072}{2835 h^{6}}\left(x-x_{n}\right)^{8}-\frac{2048}{25515 h^{6}}\left(x-x_{n}\right)^{9}\right] f_{n+\frac{3}{4}} \\
& +\left[-\frac{117415 h}{241920}\left(x-x_{n}\right)+\frac{270}{36 h}\left(x-x_{n}\right)^{3}-\frac{1413}{72 h^{2}}\left(x-x_{n}\right)^{4}+\frac{2787}{120 h^{3}}\left(x-x_{n}\right)^{5}\right. \\
& \left.-\frac{1333}{90 h^{4}}\left(x-x_{n}\right)^{6}+\frac{331}{63 h^{5}}\left(x-x_{n}\right)^{7}-\frac{41}{42 h^{6}}\left(x-x_{n}\right)^{8}+\frac{2}{27 h^{6}}\left(x-x_{n}\right)^{9}\right] f_{n+1} \\
& +\left[\frac{73279 h}{544320}\left(x-x_{n}\right)-\frac{180}{81 h}\left(x-x_{n}\right)^{3}+\frac{501}{81 h^{2}}\left(x-x_{n}\right)^{4}-\frac{1066}{135 h^{3}}\left(x-x_{n}\right)^{5}\right. \\
& \left.+\frac{2214}{405 h^{4}}\left(x-x_{n}\right)^{6}-\frac{1184}{567 h^{5}}\left(x-x_{n}\right)^{7}+\frac{78}{189 h^{6}}\left(x-x_{n}\right)^{8}-\frac{8}{243 h^{6}}\left(x-x_{n}\right)^{9}\right] f_{n+\frac{3}{2}} \\
& +\left[-\frac{53323 h}{1209600}\left(x-x_{n}\right)+\frac{135}{180 h}\left(x-x_{n}\right)^{3}-\frac{774}{360 h^{2}}\left(x-x_{n}\right)^{4}+\frac{1713}{600 h^{3}}\left(x-x_{n}\right)^{5}\right. \\
& \left.-\frac{187}{90 h^{4}}\left(x-x_{n}\right)^{6}+\frac{265}{315 h^{5}}\left(x-x_{n}\right)^{7}-\frac{37}{210 h^{6}}\left(x-x_{n}\right)^{8}+\frac{2}{135 h^{6}}\left(x-x_{n}\right)^{9}\right] f_{n+2} \\
& +\left[\frac{5989 h}{6048000}\left(x-x_{n}\right)-\frac{54}{315 h}\left(x-x_{n}\right)^{3}+\frac{45}{90 h^{2}}\left(x-x_{n}\right)^{4}-\frac{51}{75 h^{3}}\left(x-x_{n}\right)^{5}\right. \\
& \left.+\frac{23}{45 h^{4}}\left(x-x_{n}\right)^{6}-\frac{68}{315 h^{5}}\left(x-x_{n}\right)^{7}+\frac{5}{105 h^{6}}\left(x-x_{n}\right)^{8}-\frac{4}{9455 h^{6}}\left(x-x_{n}\right)^{9}\right] f_{n+\frac{5}{2}} \\
& +\left[-\frac{34543 h}{32659200}\left(x-x_{n}\right)+\frac{90}{4860 h}\left(x-x_{n}\right)^{3}-\frac{531}{9720 h^{2}}\left(x-x_{n}\right)^{4}+\frac{1223}{16200 h^{3}}\left(x-x_{n}\right)^{5}\right. \\
& \left.-\frac{141}{2430 h^{4}}\left(x-x_{n}\right)^{6}+\frac{215}{8505 h^{5}}\left(x-x_{n}\right)^{7}-\frac{33}{5670 h^{6}}\left(x-x_{n}\right)^{8}+\frac{2}{3645 h^{6}}\left(x-x_{n}\right)^{9}\right] f_{n+3}
\end{aligned}
$$

Evaluating (3.7) at $x=x_{n+j} j=\frac{3}{4}, 1, \frac{3}{2}, 2, \frac{5}{2}, 3$ and the first derivative of Equation (3.7) at $x=x_{n}$, to obtain the following discrete schemes to form our block method. 


$$
\begin{aligned}
& y_{n+\frac{3}{4}}-\frac{3}{2} y_{n+\frac{1}{2}}+\frac{1}{2} y_{n}=\frac{329909}{4644860} h^{2} f_{n}+\frac{1464259}{10321920} h^{2} f_{n+\frac{1}{2}}-\frac{125471}{1088640} h^{2} f_{n+\frac{3}{4}}+\frac{774913}{10321920} h^{2} f_{n+1} \\
& -\frac{941669}{46448640} h^{2} f_{n+\frac{3}{2}}+\frac{16889}{2580480} h^{2} f_{n+2}-\frac{15049}{10321920} h^{2} f_{n+\frac{5}{2}}+\frac{43163}{278691840} h^{2} f_{n+3} \\
& y_{n+1}-2 y_{n+\frac{1}{2}}+y_{n}=\frac{2057}{145152} h^{2} f_{n}+\frac{111659}{40320} h^{2} f_{n+\frac{1}{2}}-\frac{304}{1701} h^{2} f_{n+\frac{3}{4}}+\frac{4177}{26880} h^{2} f_{n+1} \\
& -\frac{7339}{181440} h^{2} f_{n+\frac{3}{2}}+\frac{1051}{80640} h^{2} f_{n+2}-\frac{13}{4480} h^{2} f_{n+\frac{5}{2}}+\frac{671}{2177280} h^{2} f_{n+3} \\
& y_{n+\frac{3}{2}}-3 y_{n+\frac{1}{2}}+2 y_{n}=\frac{20341}{725760} h^{2} f_{n}+\frac{23861}{40320} h^{2} f_{n+\frac{1}{2}}-\frac{2792}{8505} h^{2} f_{n+\frac{3}{4}}+\frac{39343}{80640} h^{2} f_{n+1} \\
& -\frac{8437}{181440} h^{2} f_{n+\frac{3}{2}}+\frac{16889}{2580480} h^{2} f_{n+2}-\frac{29}{5760} h^{2} f_{n+\frac{5}{2}}+\frac{1187}{2177280} h^{2} f_{n+3} \\
& y_{n+2}-4 y_{n+\frac{1}{2}}+3 y_{n}=\frac{3049}{72576} h^{2} f_{n}+\frac{3599}{4032} h^{2} f_{n+\frac{1}{2}}-\frac{4064}{8505} h^{2} f_{n+\frac{3}{4}}+\frac{34163}{40320} h^{2} f_{n+1} \\
& +\frac{13333}{90720} h^{2} f_{n+\frac{3}{2}}+\frac{2299}{40320} h^{2} f_{n+2}-\frac{181}{20160} h^{2} f_{n+\frac{5}{2}}+\frac{991}{1088640} h^{2} f_{n+3} \\
& y_{n+\frac{5}{2}}-5 y_{n+\frac{1}{2}}+4 y_{n}=\frac{20149}{362880} h^{2} f_{n}+\frac{8087}{6720} h^{2} f_{n+\frac{1}{2}}-\frac{5584}{8505} h^{2} f_{n+\frac{3}{4}}+\frac{9955}{8064} h^{2} f_{n+1} \\
& +\frac{6367}{18144} h^{2} f_{n+\frac{3}{2}}+\frac{4057}{13440} h^{2} f_{n+2}+\frac{197}{20160} h^{2} f_{n+\frac{5}{2}}+\frac{611}{1088640} h^{2} f_{n+3} \\
& y_{n+3}-6 y_{n+\frac{1}{2}}+5 y_{n}=\frac{52103}{725760} h^{2} f_{n}+\frac{58657}{40320} h^{2} f_{n+\frac{1}{2}}-\frac{5584}{8505} h^{2} f_{n+\frac{3}{4}}+\frac{23173}{16128} h^{2} f_{n+1} \\
& +\frac{23987}{36288} h^{2} f_{n+\frac{3}{2}}+\frac{40657}{80640} h^{2} f_{n+2}+\frac{10529}{40320} h^{2} f_{n+\frac{5}{2}}+\frac{36637}{2177280} h^{2} f_{n+3} \\
& y_{n}^{\prime}=-\frac{1}{32659200 h}\left[65318400 y_{n}-65318400 y_{n+\frac{1}{2}}+3347511 h^{2} f_{n}+15851025 h^{2} f_{n+1}+1439721 h^{2} f_{n+2}\right. \\
& \left.+34543 h^{2} f_{n+3}+16915122 h^{2} f_{n+\frac{1}{2}}-4396740 h^{2} f_{n+\frac{3}{2}}-24702976 h^{2} f_{n+\frac{3}{4}}-323406 h^{2} f_{n+\frac{5}{2}}\right]
\end{aligned}
$$

Equation (3.8) is our proposed uniform eighth order block method with the error constants exhibited in Table 1.

Table 1. Order and error constants of schemes (3.8).

\begin{tabular}{ccc}
\hline Schemes & Order & Error constants \\
\hline$y_{n+\frac{3}{4}}$ & 8 & $-\frac{1367003}{5073430118400}$ \\
$y_{n+1}$ & 8 & $-\frac{569}{1061683200}$ \\
$y_{n+\frac{3}{2}}$ & 8 & $\frac{-703}{707788800}$ \\
$y_{n+2}$ & 8 & $-\frac{631}{412876800}$ \\
$y_{n+\frac{5}{2}}$ & 8 & $-\frac{2671}{1486356480}$ \\
$y_{n+3}$ & 8 & $-\frac{1859}{495452160}$ \\
$y_{n}^{\prime}$ & 8 & $\frac{126297}{2048}$ \\
\hline
\end{tabular}


Also the first derivative of (3.7) is evaluated at $x=x_{n+j} j=\frac{1}{2}, \frac{3}{4}, 1, \frac{3}{2}, 2, \frac{5}{2}, 3$ as follows

$$
\begin{aligned}
y_{n+\frac{1}{2}}^{\prime}= & \frac{1}{32659200 h}\left[-65318400 y_{n}+65318400 y_{n+\frac{1}{2}}+939729 h^{2} f_{n}+10585755 h^{2} f_{n+1}+899559 h^{2} f_{n+2}\right. \\
& \left.+21197 h^{2} f_{n+3}+16257618 h^{2} f_{n+\frac{1}{2}}-2802180 h^{2} f_{n+\frac{3}{2}}-17537024 h^{2} f_{n+\frac{3}{4}}-199854 h^{2} f_{n+\frac{5}{2}}\right] \\
y_{n+\frac{3}{4}}^{\prime}= & \frac{1}{4180377600 h}\left[-8360755200 y_{n}+8360755200 y_{n+\frac{1}{2}}+117608847 h^{2} f_{n}+1161584685 h^{2} f_{n+1}\right. \\
& +104563737 h^{2} f_{n+2}+2485771 h^{2} f_{n+3}+2489303394 h^{2} f_{n+\frac{1}{2}} \\
& \left.-321829620 h^{2} f_{n+\frac{3}{2}}-1440166912 h^{2} f_{n+\frac{3}{4}}-23361102 h^{2} f_{n+\frac{5}{2}}\right] \\
y_{n+1}^{\prime}= & \frac{1}{32659200 h}\left[-65318400 y_{n}+65318400 y_{n+\frac{1}{2}}+927849 h^{2} f_{n}+13321935 h^{2} f_{n+1}+880119 h^{2} f_{n+2}\right. \\
& \left.+20657 h^{2} f_{n+3}+19061838 h^{2} f_{n+\frac{1}{2}}-2768700 h^{2} f_{n+\frac{3}{2}}-6754304 h^{2} f_{n+\frac{3}{4}}-194994 h^{2} f_{n+\frac{5}{2}}\right] \\
y_{n+\frac{5}{2}}^{\prime}= & \frac{1}{32659200 h}\left[-65318400 y_{n}+65318400 y_{n+\frac{1}{2}}+801489 h^{2} f_{n}+31736475 h^{2} f_{n+1}+22050279 h^{2} f_{n+2}\right. \\
y_{n+\frac{3}{2}}^{\prime}= & \frac{1}{32659200 h}\left[-65318400 y_{n}+65318400 y_{n+\frac{1}{2}}+880689 h^{2} f_{n}+27057915 h^{2} f_{n+1}+134919 h^{2} f_{n+2}\right. \\
& \left.+9197 h^{2} f_{n+3}+20482578 h^{2} f_{n+\frac{1}{2}}+4950780 h^{2} f_{n+\frac{3}{2}}-12621824 h^{2} f_{n+\frac{3}{4}}-70254 h^{2} f_{n+\frac{5}{2}}\right] \\
& \left.+42257 h^{2} f_{n+3}+18750798 h^{2} f_{n+\frac{1}{2}}+17483460 h^{2} f_{n+\frac{3}{2}}-6754304 h^{2} f_{n+\frac{3}{4}}-506034 h^{2} f_{n+\frac{5}{2}}\right] \\
y_{n+2}^{\prime}= & \frac{1}{32659200 h}\left[-65318400 y_{n}+65318400 y_{n+\frac{1}{2}}+949449 h^{2} f_{n}+19814895 h^{2} f_{n+1}+7373079 h^{2} f_{n+2}\right. \\
& -65318400 y_{n}+65318400 y_{n+\frac{1}{2}}+1434729 h^{2} f_{n}-12701745 h^{2} f_{n+1}+1709559 h^{2} f_{n+2} \\
& \left.+8279118 h^{2} f_{n+\frac{1}{2}}+36122820 h^{2} f_{n+\frac{3}{2}}+24702976 h^{2} f_{n+\frac{3}{4}}+25517646 h^{2} f_{n+\frac{5}{2}}\right]
\end{aligned}
$$

Equation (3.9) has the following order and error constants in Table 2.

\section{Implementation Strategies}

Equation (3.9) is substituted in Equation (3.8) when applying to Equation (1.0) directly at $n=0$, simultaneously produces solutions at the point $y_{\frac{1}{2}}, y_{\frac{3}{4}}, y_{1}, y_{\frac{3}{2}}, y_{2}, y_{\frac{5}{2}}, y_{3}$ at once without any recourse to special predictor 
Table 2. Order and error constants of schemes (3.9).

\begin{tabular}{ccc}
\hline Schemes & Order & Error constants \\
\hline$y_{n+\frac{1}{2}}^{\prime}$ & 8 & $-\frac{150147}{4096}$ \\
$y_{n+\frac{3}{4}}^{\prime}$ & 8 & $-\frac{35647065}{8192}$ \\
$y_{n+1}^{\prime}$ & 8 & $-\frac{72999}{2048}$ \\
$y_{n+\frac{3}{2}}^{\prime}$ & 8 & $-\frac{95139}{4096}$ \\
$y_{n+2}^{\prime}$ & 8 & $-\frac{99495}{2048}$ \\
$y_{n+\frac{5}{2}}^{\prime}$ & 8 & $\frac{89469}{4096}$ \\
$y_{n+3}^{\prime}$ & 8 & $-\frac{713511}{2048}$ \\
\hline
\end{tabular}

for $y_{n}^{\prime}$ present in the method. For the advancement in the integration processes we used schemes derived at $y_{n+\frac{3}{4}}$, $y_{n+\frac{5}{2}}, y_{n+3}$ together as $n=1,2, \cdots$. This new method is demonstrated on linear and non linear problems to ascertain their degree of accuracy with the existing methods.

\section{Numerical Experiments}

Three numerical experiments of two linear and one non linear problem were used to ascertain the efficiency of the method.

Example 1

$$
\begin{gathered}
y^{\prime \prime}+\frac{6}{x} y^{\prime}+\frac{4}{x^{2}} y=0 \\
y(1)=1, \quad y^{\prime}(1)=1, h=\frac{0.1}{32} \quad x>0 .
\end{gathered}
$$

Theoretical solution is $y(x)=\frac{5}{3 x}-\frac{2}{3 x^{4}}$.

\section{Example 2}

$$
\begin{gathered}
y^{\prime \prime}-3 y^{\prime}=8 \mathrm{e}^{2 x} \\
y(0)=1, \quad y^{\prime}(0)=1, \quad h=0.005
\end{gathered}
$$

Theoretical solution is $y(x)=-4 \mathrm{e}^{2 x}+3 \mathrm{e}^{3 x}+2$.

Example 3

$$
\begin{gathered}
y^{\prime \prime}-3 y^{\prime}=8 \mathrm{e}^{2 x y} \\
y(0)=1, \quad y^{\prime}(0)=1, \quad h=0.005
\end{gathered}
$$

No theoretical solution.

\section{Conclusion}

We want to re-emphasize the claim made by [14] for first order schemes that when the derived schemes for var- 
ious values of $k$ are of the same order the block scheme gotten from the minimal value of $k$ performed excellently well and compared favourably with the exact solutions. This has also been established for second order schemes derived from various values of $k$ which are of the same order with three different numerical experiments tested (see Figure 1, Figure 2 and Figure 3).

Table 3 and Table 4 also display the numerical result of problem 1 and absolute errors by using various block methods of $k=4, k=5$ together with the new block method at $k=3$. Table 5 and Table 6 display the numerical result of problem 2 and absolute errors by using various block methods of $k=4, k=5$ together with the new block method at $k=3$.

Table 7 displays the approximate solution of example 3 with block methods of $k=4, k=5$ together with the

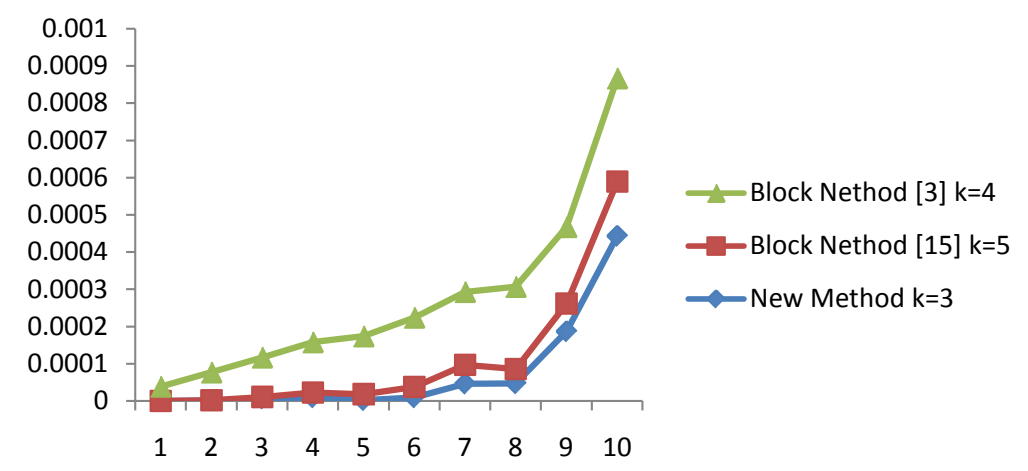

Figure 1. Error graph of problem 1.

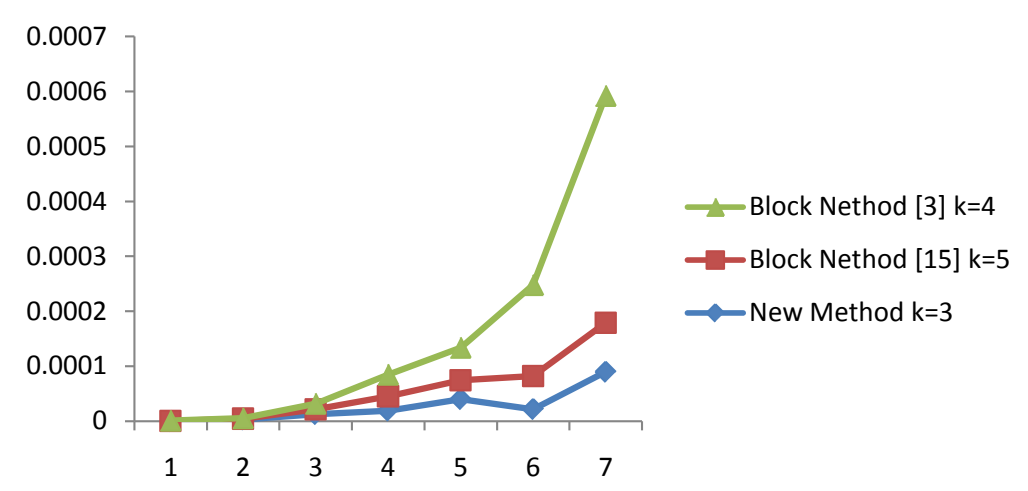

Figure 2. Error graph of problem 2.

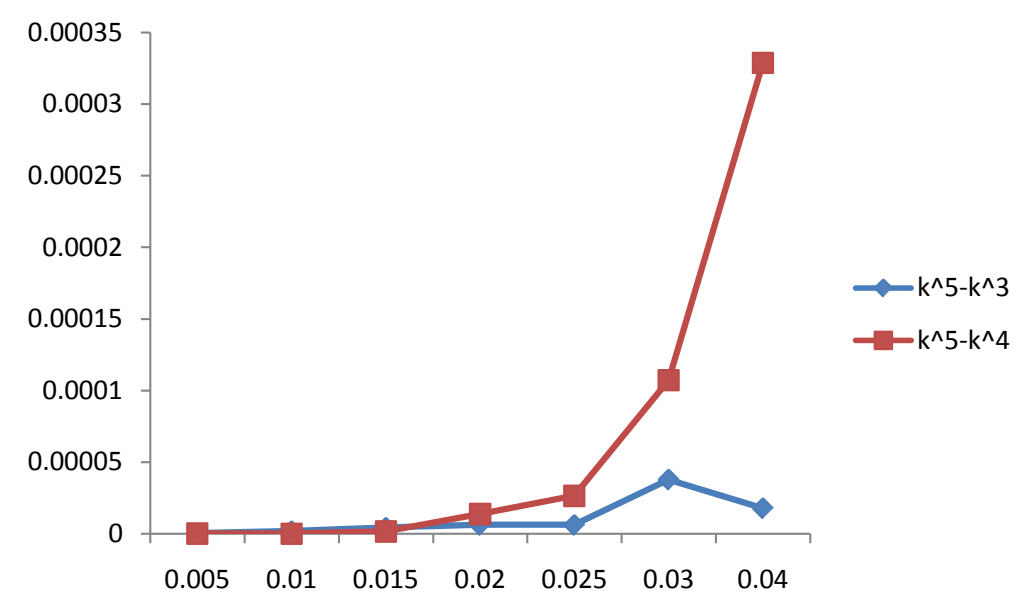

Figure 3. Error graph of problem 3. 
Table 3. Table of result for example 1.

\begin{tabular}{ccccc}
\hline$x$ & Theoretical solution & Block method [14] $k=4$ & Block method [15] $k=5$ & New block method $k=3$ \\
\hline 1.003125 & 1.003076526 & 1.003114880 & 1.0030766905 & 1.0030764430 \\
1.00625 & 1.006057503 & 1.006132507 & 1.00605684265 & 1.006055854 \\
1.009375 & 1.008944993 & 1.009050907 & 1.0089405789 & 1.008938355 \\
1.0125 & 1.011741018 & 1.011876494 & 1.01172802434 & 1.011731527 \\
1.015625 & 1.014447543 & 1.014603110 & 1.014431165439 & 1.014480080 \\
1.01875 & 1.017066494 & 1.017252866 & 1.017038197167 & 1.017057078 \\
1.021875 & 1.019599755 & 1.019795810 & 1.01954923805 & 1.019553250 \\
1.025 & 1.022049164 & 1.022270209 & 1.02201055468 & 1.0220996286 \\
1.028125 & 1.024416519 & 1.024622147 & 1.02434160973 & 1.0242295932 \\
1.03125 & 1.026703578 & 1.026981486 & 1.026557694498 & 1.027146899 \\
\hline
\end{tabular}

Table 4. Absolute error of problem 1.

\begin{tabular}{ccc}
\hline Block method [3] $k=4$ & Block method [15] $k=5$ & New method $k=3$ \\
\hline $3.8354 \mathrm{E}(-05)$ & $1.645 \mathrm{E}(-07)$ & $8.3 \mathrm{E}(-08)$ \\
$7.5004 \mathrm{E}(-05)$ & $6.6035 \mathrm{E}(-07)$ & $1.16 \mathrm{E}(-06)$ \\
$1.05926 \mathrm{E}(-04)$ & $4.4141 \mathrm{E}(-06)$ & $6.638 \mathrm{E}(-06)$ \\
$1.35476 \mathrm{E}(-04)$ & $1.299366 \mathrm{E}(-05)$ & $9.491 \mathrm{E}(-06)$ \\
$1.55567 \mathrm{E}(-04)$ & $1.6377561 \mathrm{E}(-05)$ & $1.9535 \mathrm{E}(-06)$ \\
$1.863726 \mathrm{E}(-04)$ & $2.8296833 \mathrm{E}(-05)$ & $9.416 \mathrm{E}(-06)$ \\
$1.96055 \mathrm{E}(-04)$ & $5.051695 \mathrm{E}(-05)$ & $4.6505 \mathrm{E}(-05)$ \\
$2.21045 \mathrm{E}(-04)$ & $3.860932 \mathrm{E}(-05)$ & $4.7122 \mathrm{E}(-05)$ \\
$2.0562 \mathrm{E}(-04)$ & $7.490927 \mathrm{E}(-05)$ & $1.86926 \mathrm{E}(-04)$ \\
$2.77908 \mathrm{E}(-04)$ & $1.458835 \mathrm{E}(-04)$ & $4.43321 \mathrm{E}(-04)$ \\
\hline
\end{tabular}

Table 5. Table of result for example 2.

\begin{tabular}{|c|c|c|c|c|}
\hline$x$ & Theoretical solution & Block method [3] $k=4$ & Block method [15] $k=5$ & New block method $k=3$ \\
\hline 0.005 & 1.005138526 & 1.005139114 & 1.0051388419 & 1.005138368 \\
\hline 0.01 & 1.010558242 & 1.010557205 & 1.0105569711 & 1.010555066 \\
\hline 0.015 & 1.016265444 & 1.016255068 & 1.0162567886 & 1.016252503 \\
\hline 0.02 & 1.022266643 & 1.022226977 & 1.0222407282 & 1.022247320 \\
\hline 0.025 & 1.028568067 & 1.028508035 & 1.02853411642 & 1.028527886 \\
\hline 0.03 & 1.035176665 & 1.035010659 & 1.03511676083 & 1.035154590 \\
\hline 0.04 & 1.049342284 & 1.048928801 & 1.04925342567 & 1.049432200 \\
\hline
\end{tabular}


Table 6. Absolute error of problem 2.

\begin{tabular}{ccc}
\hline Block method [3] $k=4$ & Block method [15] $k=5$ & New block method $k=3$ \\
\hline $5.8849 \mathrm{E}(-07)$ & $3.159 \mathrm{E}(-07)$ & $1.58 \mathrm{E}(-07)$ \\
$1.03675 \mathrm{E}(-06)$ & $1.2709 \mathrm{E}(-06)$ & $3.176 \mathrm{E}(-06)$ \\
$1.03759 \mathrm{E}(-05)$ & $8.6554 \mathrm{E}(-06)$ & $1.2941 \mathrm{E}(-05)$ \\
$3.95659 \mathrm{E}(-05)$ & $2.59148 \mathrm{E}(-05)$ & $1.9323 \mathrm{E}(-05)$ \\
$5.97171 \mathrm{E}(-05)$ & $3.395058 \mathrm{E}(-05)$ & $4.0181 \mathrm{E}(-05)$ \\
$1.66006 \mathrm{E}(-04)$ & $5.990417 \mathrm{E}(-05)$ & $2.2075 \mathrm{E}(-05)$ \\
$4.13483 \mathrm{E}(-04)$ & $8.885833 \mathrm{E}(-05)$ & $8.9916 \mathrm{E}(-05)$ \\
\hline
\end{tabular}

Table 7. Table of result for example 3.

\begin{tabular}{cccc}
\hline$x$ & Block method [3] $k=4$ & Block method [15] $k=5$ & New block method $k=3$ \\
\hline 0.005 & 1.005139120 & 1.0051388451 & 1.005138369 \\
0.01 & 1.010557226 & 1.0105569851 & 1.010555080 \\
0.015 & 1.016255105 & 1.01625686111 & 1.016252575 \\
0.02 & 1.02222703 & 1.0222409615 & 1.022247553 \\
0.025 & 1.028508106 & 1.0285346996 & 1.028528475 \\
0.03 & 1.035010745 & 1.035118000 & 1.035155832 \\
0.04 & 1.048928909 & 1.049257509 & 1.049436332 \\
\hline
\end{tabular}

Table 8. Global error of problem 3.

\begin{tabular}{cc}
\hline$\left|y^{5}-y^{4}\right|$ & $\left|y^{5}-y^{3}\right|$ \\
$2.749 \mathrm{E}(-07)$ & $4.761 \mathrm{E}(-07)$ \\
$2.409 \mathrm{E}(-07)$ & $1.9051 \mathrm{E}(-06)$ \\
$1.75611 \mathrm{E}(-05)$ & $4.28611 \mathrm{E}(-06)$ \\
$1.39315 \mathrm{E}(-05)$ & $6.5915 \mathrm{E}(-06)$ \\
$2.65936 \mathrm{E}(-05)$ & $6.2246 \mathrm{E}(-06)$ \\
$1.07255 \mathrm{E}(-04)$ & $3.7832 \mathrm{E}(-05)$ \\
$3.286 \mathrm{E}(-04)$ & $1.78823 \mathrm{E}(-04)$ \\
\hline
\end{tabular}

Where $\left|y^{5}-y^{4}\right|=$ the absolute difference between approximate solution of $k=5$ and $k=4 ;\left|y^{5}-y^{3}\right|=$ the absolute difference between approximate solution of $k=5$ and $k=3$.

new block method at $k=3$ while Table 8 is the global or approximate error of problem 3 , since this problem has no theoretical solution.

\section{References}

[1] Yahaya, Y.A. and Badmus, A.M. (2009) A Class of Collocation Methods for General Second Order Ordinary Differential Equations. African Journal of Mathematics and Computer Science Research, 2, 69-72.

[2] Badmus, A.M. and Ekpenyong, F.E. (2013) High Order Block Method for Direct Solution of General Second Order Ordinary Differential Equations. Academy Journal of Science and Engineering, Nigerian Defence Academy Kaduna, 7, 36-42. 
[3] Badmus, A.M. and Yahaya, Y.A. (2009) An Accurate Uniform Order 6 Block Method for Direct Solution of General Second Order Ordinary Differential Equations. The Pacific Journal of Science and Technology, 10, 248-254.

[4] Jator, S.N. (2007) A Class of Initial Value Methods for Direct Solution of Second Order Initial Value Problems. 4th International Conference of Applied Mathematics and Computing, Plovdiv, 12-18 August 2007.

[5] Lambert, J.D. (1973) Computational Methods in Ordinary Differential Equations. John Wiley and Sons, New York, 278.

[6] Lambert, J.D. (1991) Numerical Method for Ordinary Differential Systems. John Wiley and Sons, New York, 293.

[7] Awoyemi, D.O. (1999) A Class of Continuous Method for General Second Order IVPs in Ordinary Differential Equations. International Journal of Computer Mathematics, 72, 29-37. http://dx.doi.org/10.1080/00207169908804832

[8] Jator, S.N. (2001) Improvements in Adams-Moulton Methods for the First Initial Value Problems. Journal of the Tennessee Academy of Science, 76, 57-60.

[9] Onumanyi, P., Awoyemi, D.O., Jator, S.N. and Siriseria, U.W. (1994) New Linear Multistep Methods with Continuous Coefficients for First Order IVPs. Journal of Nigeria Mathematics Society, 13, 37-51.

[10] Awoyemi, D.O. (2003) A P-Stable Linear Multistep Method for Direct Solution of General Third Order Ordinary Differential Equation. International Journal of Computer Mathematics, 80, 987-993. http://dx.doi.org/10.1080/0020716031000079572

[11] Vigo-Angular, J. and Ramos, H. (2006) Variable Step-Size Implementation of Multi-Step Methods $y^{\prime \prime}=f\left(x, y, y^{\prime}\right)$. Journal of Computation and Applied Mathematics, 92, 114-131. http://dx.doi.org/10.1016/j.cam.2005.04.043

[12] Fatunla, S.O. (1991) Block Method for Second Order Differential Equations. International Journal of Computer Mathematics, 41, 55-63. http://dx.doi.org/10.1080/00207169108804026

[13] Yahaya, Y.A. (2004) Some Theories and Applications of Continuous LMM for Ordinary Differential Equations. PhD Thesis (Unpublished), University of Jos, Nigeria.

[14] Badmus, A.M. and Mshelia, D.W. (2012) Uniform Order Zero Stable k-Step Block Methods for Initial Value Problems of Ordinary Differential Equations. Journal of Nigerian Association of Mathematical Physics, 20, 65-74.

[15] Badmus, A.M. (2014) An Efficient Seven Point Block Method for Direct Solution of General Second Order Ordinary Differential Equations $y^{\prime \prime}=f\left(x, y, y^{\prime}\right)$. British Journal of Mathematics and Computer Science, 4, 2840-2852.

http://dx.doi.org/10.9734/BJMCS/2014/6749 
Scientific Research Publishing (SCIRP) is one of the largest Open Access journal publishers. It is currently publishing more than 200 open access, online, peer-reviewed journals covering a wide range of academic disciplines. SCIRP serves the worldwide academic communities and contributes to the progress and application of science with its publication.

Other selected journals from SCIRP are listed as below. Submit your manuscript to us via either submit@scirp.org or Online Submission Portal.
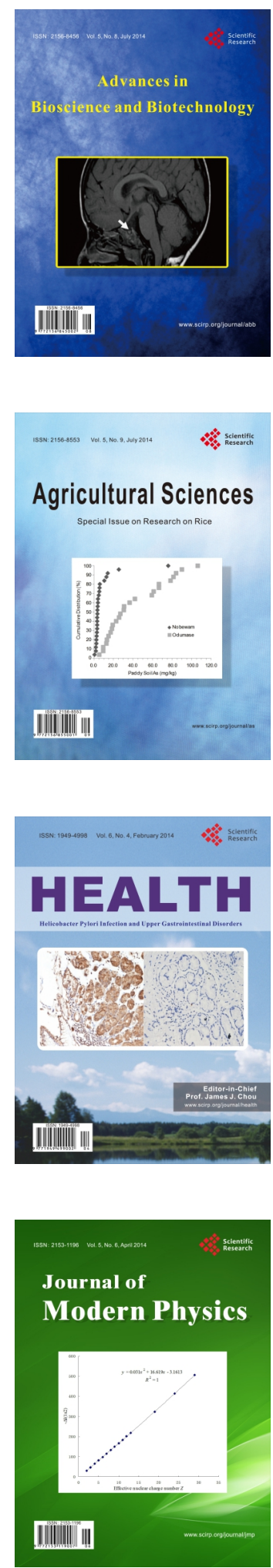
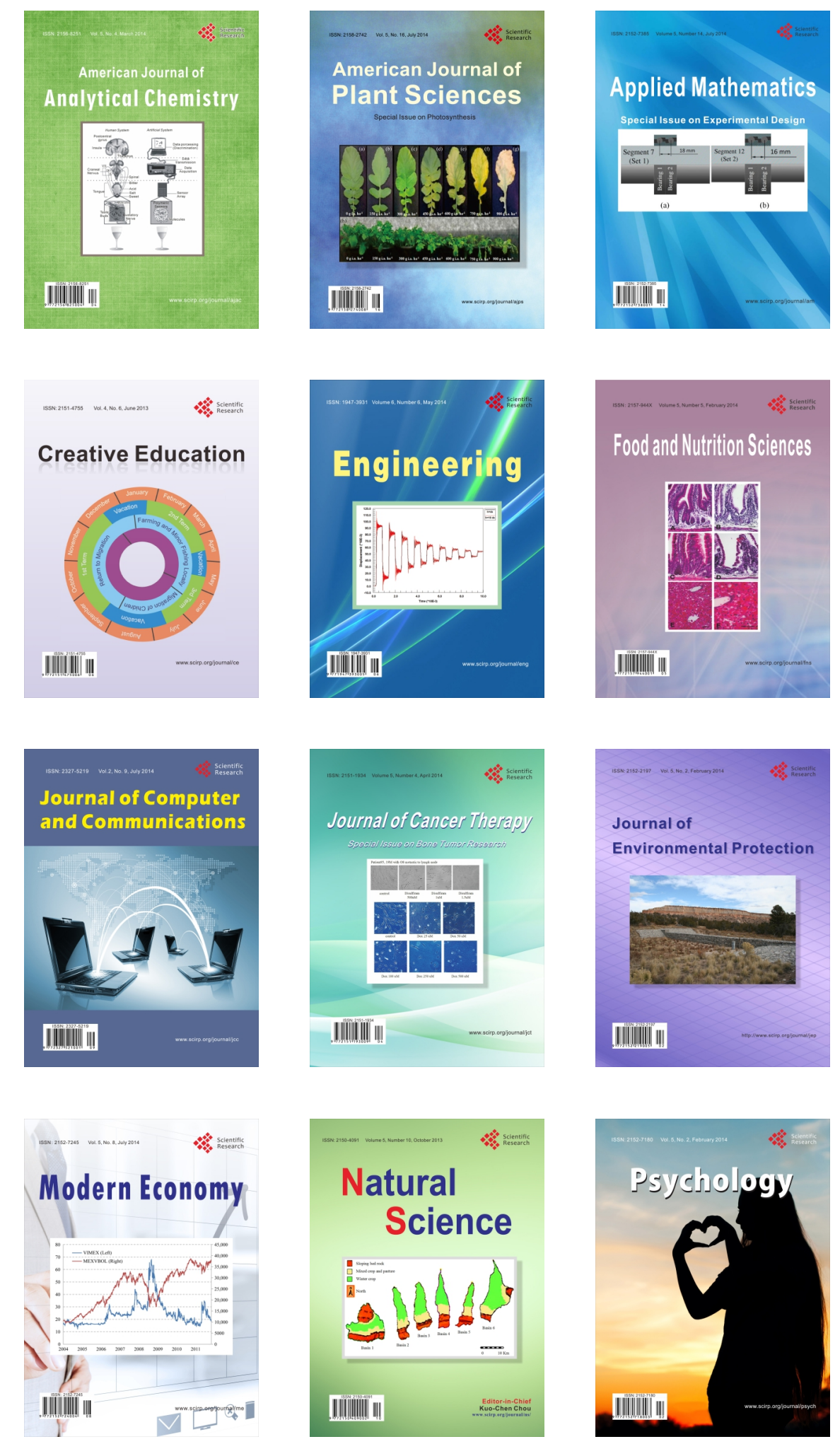